\title{
Active Matter
}

\author{
Ramin Golestanian ${ }^{1}$ and Sriram Ramaswamy ${ }^{2}$ \\ 1 Rudolf Peierls Centre for Theoretical Physics, University of Oxford, Oxford, UK \\ ${ }^{2}$ Indian institute of Science, Bangalore, India
}

Received 3 June 2013

Published online: 28 June 2013 - (C) EDP Sciences / Società Italiana di Fisica / Springer-Verlag 2013

The living cell and many of its components, life-forms large and small, and artificially energized mimics of motility are all examples of Active Matter, in which free energy is taken up and consumed at the level of each particle, generally leading to systematic movement. An understanding of the individual and collective behaviour of active particles is one of the grand challenges of nonequilibrium statistical physics, and holds the key to a physical grasp of the mechanics and statistics of living matter. It is now widely appreciated that mechanics, through the exertion, detection, modulation and transduction of forces, plays a crucial role in cellular processes, decisions and fate. Fluctuations, flow, instabilities, and ordering phase transitions are seen over an enormous range of length scales in the living world. The success of agent-based flocking models, theories of the swimming of individual microorganisms, fundamental experimental and theoretical advances in catalytic self-propulsion, hydrodynamic theories of self-driven fluids, and increasingly quantitative experiments on extracts, cells and organisms testify to the extraordinary ferment created by the rise of active-matter physics. For continued intellectual vitality it is essential from time to time for a field to take stock, consolidate, and identify new challenges, new unifying principles, and the most promising directions for progress. We believe Active Matter is now at such a stage. The unique strength of the European Physical Journal E lies in its commitment to the physics of soft matter and biological systems, and we wish to take this opportunity to move that young field forward. Accordingly, this Topical Issue of EPJE presents the latest from some very active participants in this exciting field. In this issue you will find articles on: individual swimmers, modelled theoretically in Stokesian fluids [1-6], simulated in molecularly detailed biomembranes [7], and realized in the form of catalytic colloids [8]; cilia and flagella, beating individually [9,10], magnetically actuated [11], or synchronizing [12]; the collective behaviour of suspensions of active filaments, in experiments on cell extracts [13] and in hydrodynamic treatments [1419], highlighting the roles of order, instabilities, chirality, concentration and condensation; anomalous spatiotemporal distributions arising from active motion [20,21]; and self-propulsion on solid surfaces, in theory [22] and in vibratedgrain experiments [23]. We are confident that these outstanding articles will stimulate new progress in the study of the dynamics and organization of self-driven particles, in charta, in silico, in vitro and in vivo, and we are delighted to offer this special issue to our readers.

\section{References}

1. A. Pandey, R.A. Simha, Eur. Phys. J. E 35, 52 (2012).

2. R. Ledesma-Aguilar, H. Löwen, J.M. Yeomans, Eur. Phys. J. E 35, 70 (2012).

3. E. Passov, Y. Or, Eur. Phys. J. E 35, 78 (2012).

4. M. Leoni, T.B. Liverpool, Eur. Phys. J. E 35, 126 (2012).

5. G. Oukhaled, A. Cebers, J.-C. Bacri, J.-M. Di Meglio, C. Py, Eur. Phys. J. E 35, 121 (2012).

6. A. Zöttl, H. Stark, Eur. Phys. J. E 36, 4 (2013).

7. M.-J. Huang, H.-Y. Chen, A.S. Mikhailov, Eur. Phys. J. E 35, 119 (2012).

8. W. Duan, M. Ibele, R. Liu, A. Sen, Eur. Phys. J. E 35, 77 (2012).

9. A. Vilfan, Eur. Phys. J. E 35, 72 (2012).

10. T.D. Montenegro-Johnson, A.A. Smith, D.J. Smith, D. Loghin, J.R. Blake, Eur. Phys. J. E 35, 111 (2012).

11. P. Tierno, F. Sagues, Eur. Phys. J. E 35, 71 (2012).

12. N. Uchida, R. Golestanian, Eur. Phys. J. E 35, 135 (2012).

13. V. Schaller, B. Hammerich, A.R. Bausch, Eur. Phys. J. E 35, 81 (2012). 
14. G. Foffano, J.S. Lintuvuori, A.N. Morozov, K. Stratford, M.E. Cates, D. Marenduzzo, Eur. Phys. J. E 35, 98 (2012).

15. N. Desreumaux, N. Florent, E. Lauga, D. Bartolo, Eur. Phys. J. E 35, 68 (2012).

16. S. Fürthauer, M. Strempel, S.W. Grill, F. Jülicher, Eur. Phys. J. E 35, 89 (2012).

17. A. Baskaran, M.C. Marchetti, Eur. Phys. J. E 35, 95 (2012).

18. N. Sarkar, A. Basu, Eur. Phys. J. E 35, 115 (2012).

19. J.-F. Joanny, K. Kruse, J. Prost, S. Ramaswamy, Eur. Phys. J. E 36, 52 (2013).

20. K. Martens, L. Angelani, R. Di Leonardo, L. Bocquet, Eur. Phys. J. E 35, 84 (2012).

21. B. Eckhardt, S. Zammert, Eur. Phys. J. E 35, 96 (2012).

22. A. DeSimone, A. Tatone, Eur. Phys. J. E 35, 85 (2012).

23. V. Yadav, A. Kudrolli, Eur. Phys. J. E 35, 104 (2012). 